\title{
Sleep Coach: A home-cared sleep doctor
}

\author{
Rayleigh Ping-Ying Chiang \\ Department of Otolaryngology Head \& Neck Surgery, \\ Shin Kong Wu Ho Su Memorial Hospital \\ Taipei, Taiwan \\ Department of Otolaryngology Head \& Neck Surgery, \\ School of Medicine, Fu Jen catholic University, \\ Taipei, Taiwan \\ Sleep Technology Special Interest Group, INSIGHT \\ Center, National Taiwan University, \\ Taipei, Taiwan.
}

\author{
Shih-Chung Kang, Chia-Hsuan Chiang, I-Ling Chen \\ Yi Chen, Jhe-Wun Yang \\ Department of Civil Engineering \\ National Taiwan University \\ Taipei, Taiwan
}

\author{
Zai-Ting Yeh, Peng-Chih Wang \\ Department of Clinical Psychology \\ Fu Jen Catholic University \\ Taipei County, Taiwan
}

\begin{abstract}
A prototype of Sleep Coach was developed to resolve long-lasting problems of cognitive behavioral therapy for insomnia patients: low compliance and low levels of patient feedback. Sleep Coach, designed to function like an ordinary book, provides a diary module to remind patients to log sleeprelated activities and a training module for helping patients practice relaxation techniques. From the tests in a sleep lab, we found users will naturally work with Sleep Coach, and that use of the training module can result in a significantly reduced pulse rate, which indicates better relaxation.
\end{abstract}

Keywords-insomnia; home-based Cognitive Behavioral Therapy

\section{INTRODUCTION}

Insomnia is a highly prevalent complaint among the general population in modern society. According to one epidemiological study[1], about one-third of the adult population exhibit at least one symptom of insomnia. Furthermore, in this population, an estimated 6\% of adult's symptoms meet the diagnosis criteria of insomnia. Insomnia is defined as repeated sleep difficulties, such as difficulty with sleep initiation, maintenance or quality that occurs during adequate sleep time and opportunity which typically leads to some form of daytime impairment [2]. Insomnia can adversely affect different aspects of an individual, such as health, quality of life and occupational or academic performance. Furthermore, the risks of traffic or work-site accidents and psychiatric disorders may increase. Pharmacotherapy is efficacious on situational insomnia which is often caused by acute stress. However, it is not efficacy for chronic insomnia and may cause several side-effects, such as tolerance and dependence when used over a long period.

Recently, the efficacy of CBT has been proven in many studies [3]. It is aimed at maladaptive sleep habits, autonomic and cognitive arousal, dysfunctional beliefs and attitudes about sleep which are believed to cause chronic insomnia. The most frequently used CBT therapies for chronic insomnia are stimulus control, sleep restriction, sleep hygiene, relaxation training, and cognitive therapy. One study has proven that CBT treatment can help improve the sleep difficulties of 70 $80 \%$ of insomnia sufferers [4].

However, CBT takes time to prove its efficacy and it combines several instructions which are different for each one, thus these characteristics of CBT decrease the compliance of patient. The average duration of CBT for once is between 6 to 10 weeks, so that the patients would not get improvement from CBT immediately. Moreover, CBT combines several instructions together, thus the patients are easy to get confused with them and then might be frustrated when applying these instructions. For example, they might forget how to practice the relaxation techniques, even though they had learned it in the hospital and felt frustrated when they were trying to practice it at home.

On the other hands, the clinical professionals do not have viable instruments to monitor the patient's compliance and practicing performance. All they could do is to trust their patient based on their self reports and these reports might not represent the real situation of them. In order to resolve these difficulties of applying a CBT therapy, we were trying to develop a home-based sleep-aided device, Sleep Coach, to help insomniacs increase their compliance and help clinical professionals monitor their patients' performance and then arrange better treatment plans for them.

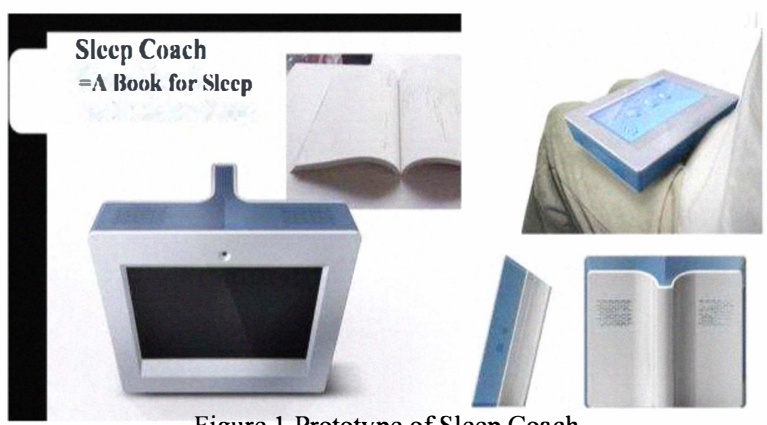

Figure 1 Prototype of Sleep Coach

\section{SLEEP COACH}

Sleep Coach is a live prototype, which is designed to function like an ordinary book (shown in Fig. 1). The design concept is based on observation of users' behavior and their bedroom environment. Most people would read a book before sleeping. Moreover, the image of book is easy to fit into a bedroom setting. The process of developing Sleep Coach is shown in Fig. 2. 


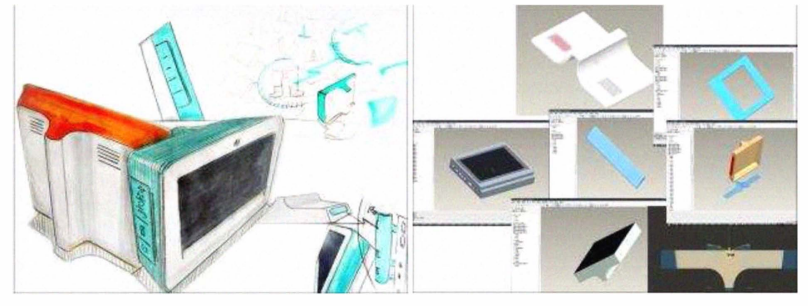

Figure 2 The process of developing of Sleep Coach

Sleep Coach provides a training module for helping patients practice relaxation techniques and a diary module to remind patients to $\log$ sleep-related activities. It also provides a screening module with a brief Insomnia Screening Scale (BISS-C)[5] designed to help patients evaluate the severity of their insomniac symptoms. Moreover, the advice module is to help the patient remember the details of their treatment plans. The main purpose of Sleep Coach is to promote the cooperation between insomnia patients and physicians. The main modules of Sleep Coach are shown in Fig. 3.

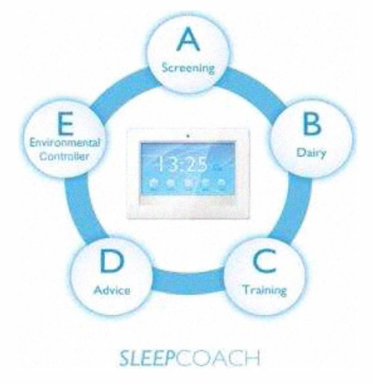

Figure 3 Main modules in Sleep Coach

\section{Training Module of Sleep Coach for Reminding}

The patients could always have Sleep Coach with them to offer home-based training programs and provide (or repeat) the advice of the physician. They would learn the relaxation technique in the hospital and then can practice it at home following the instructions, diffuse glow, of training module in Sleep Coach. Moreover, they can get real-time feedback based on their heart rate. For the physicians, they could monitor the patients training performance based on the data from this modules. The interface of training modules is shown in Fig. 4.

\section{Dairy Module of Sleep Coach for Monitoring}

The patients are able to prepare their personal data and sleep diary before going to the hospital (shown in Fig. 4). Once at the hospital, the physician can get the whole profile by reviewing the information of Sleep Coach. The patients can receive the suggestions at the first meeting with the physicians and carry out treatment plans with Sleep Coach's monitoring the situation. This can significantly reduce the time of the entire diagnosis process. The quality of treatment can also be improved due to the provision of more precise information.

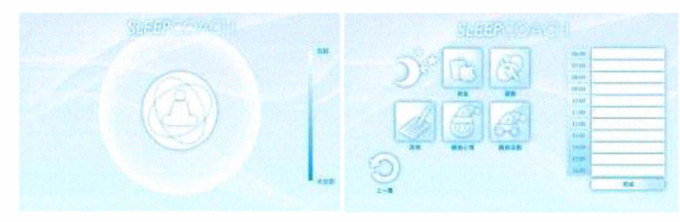

Figure 4 Interfaces of Dairy Modules in Sleep Coach

\footnotetext{
Identify applicable sponsor/s here. (sponsors)
}

\section{Validation Research of Sleep Coach}

We invited 5 participants to evaluate the validity of training tasks in Sleep Coach. All participants have to practice two tasks including a visualized signal breathing task (SB) and a hearing guide imaging task (GI) with measuring their pulse rate at the same time. From the tests in a sleep lab, we found users will naturally work with Sleep Coach, and that use of the training module can result in a significantly reduced pulse rate (shown in Fig. 5), which indicates better relaxation.

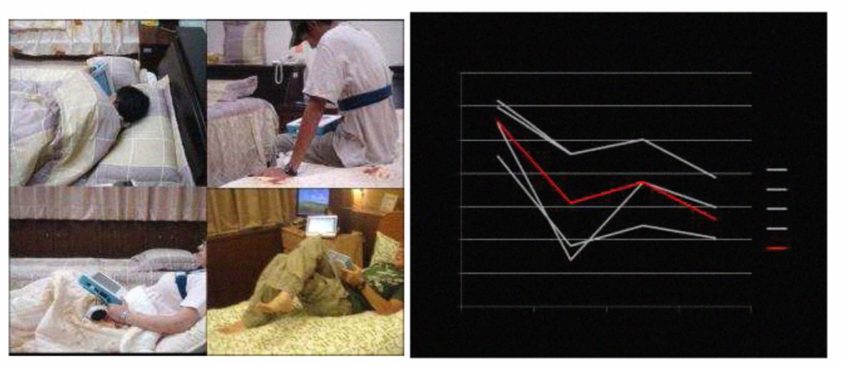

Figure 5 Pulse rate of Participants in validation research.

\section{Demonstration in Pervasive Health 2010}

Interaction would become the major point which we plan to get. We would invite the visitors to experience the major components in Sleep Coach: the training and diary modules. If there were too many visitors, we will invite one of them to be a user who would be asked to follow the instructions of the training modules to relax with earphone. Meanwhile, we will project the training modules interface on the wall. Thus we can introduce the entire workflow of Sleep Coach to the audience and the audience will see the whole process and feedbacks while the user is practicing the task at the same time. At the end of the trial, we will invite the user to share his or her feeling about Sleep Coach. The demo can be run at anytime. It takes six minutes to conduct a trial. The setting of demonstration is shown in Fig. 6.

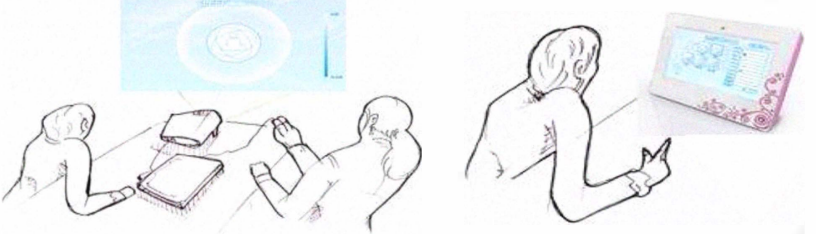

Figure 6 The setting of demonstration

\section{REFERENCES}

M. M. Ohayon, "Epidemiology of insomnia: what we know and what we still need to learn," Sleep Medicine Reviews, vol. 6, pp. 97-111, 2002.

[2] American Academy of Sleep Medicine, International Classification of sleep Disorders, 2ed : Diagnostic \& Coding Manual., Second ed. Westchester, Illinois: American Academy of Sleep Medicine, 2005.

[3] C. M. Morin, "Cognitive-behavioral Therapy of Insomnia," Sleep medicine clinics, vol. 1, pp. 375 - 386, 2006.

[4] C. M. Morin, P. J. Hauri, C. A.Espie, A. J. Spielman, D. J. Buysse, and R. R. Bootzin, "Nonpharmacologic Treatment Of Chronic Insomnia," Sleep, vol. 22, pp. 1134-1156, 1999.

[5] R. P. Y. Chiang and Z. T. Yeh, "Development of the Brief Insomnia Screening Scale in Chinese (BISS-C)Based on ICSD-II Criteria: a Pilot Study," in The 6th Congress of Asian Sleep Research Society(ASRS) Osaka, Japan, 2009. 